\title{
Quality of Online Courses
}

\author{
Afsaneh Sharif ${ }^{a}$ \\ Data de defensa: 30/06/2014
}

Palabras clave: quality assurance, online program, instructional designer.

\begin{abstract}
Instructional designers strive to create quality online courses by using different methods and strategies; however, they rarely find the time to reflect on their work and evaluate their final course design. There are three main challenges that instructional designers face while developing online course. First, instructional designers have ambiguity around their roles, and their skills and expertise are often not used as they fulfill their responsibilities. Second, despite the existence of different guidelines and procedures developed for quality assurance, there are no specific and descriptive steps that an instructional designer can complete to arrive at a goodquality course. This is because each designer may define each step or element differently, and given the fact that these steps/phases are not defined and explained thoroughly, they are taken differently. Third, constant changes with technology and online learning often place the instructional designers in unfamiliar situations. These three challenges require instructional designers to be responsive to everyday shifting demands and to continuously update their skills and knowledge. This study takes a comprehensive look at the key elements for quality online courses within the field of instructional design by examining the core elements of effective design in existing guidelines and benchmarks. The research also explores key factors for a quality online course in different phases of the course development process from instructional designers' perspectives. To gain a better understanding of the ways in which designers approach their work and strive for a good-quality result, different research methods were used in this study. To answer the research questions, I used a quantitative approach, which included surveys in different steps and locations, was used to gather the elements that 52 designers focus on and find critical in their quality design. The surveys were conducted in both Spain and Canada to examine the impact of culture on how instructional designers see the core elements of design. A
\end{abstract}

\footnotetext{
a University of British Columbia - EUA.
} 
qualitative approach, an interview, was the main focus of this study and was used to explore the views of a good-quality course, examining the backgrounds and experiences of eight instructional designers and their views of "an ideal course". The instructional designers in this research agreed that having a guideline or a policy was important, which is supported by other professionals in the field. At the same time they all mentioned that a rigid and fixed guideline might not be welcome and practical in an institution. They argued that the field of instructional design is changing as technology is evolving; therefore, the standards and guidelines developed today for a quality online course might be irrelevant tomorrow. As a result, a flexible descriptive quality framework for instructional design seemed to be what instructional designers needed in their online course development, which is supported by other educators. The study andobservation of the evolution of technology, instructors and learners' roles, and designers' cultural and educational differences led to development of a flexible online course development guideline within this field. The guideline can be found at http://wiki.ubc.ca/Design_Quality_OnlineCourse. The study also provides valuable insight into online learning and how a good online course can be developed through ongoing evaluation and emphasis of key elements in each phase of course development. 